\title{
Theoretical Analysis of Doping Management
}

\author{
Amira Tandirovic Gursel', Parviz Elahi², F. Ömer Ilday², M. Sadettin Özyazıc1 ${ }^{3}$ \\ 'Electrical and Electronics Engineering, Gaziantep University, Gaziantep, 27310, Turkey \\ gursel@gantep.edu.tr \\ ${ }^{2}$ Department of Physics, Bilkent University, Ankara, 06800, Turkey \\ elahi@fen.bilkent.edu.tr, ilday@bilkent.edu.tr \\ ${ }^{3}$ Electrical and Electronics Engineering, Bahcesehir University, Istanbul, 34353, Turkey \\ sadettin.ozyazici@bahcesehir.edu.tr
}

\begin{abstract}
Two opposing requirements, such as thermal load and nonlinear effects are important limitations in rapid progress of high-power fiber laser technologies. Thermal effects, which limit the average power, can be minimized by using low-doped, longer gain fibers, whereas presence of nonlinear effects requires use of high-doped, shorter fibers to maximize the peak power. Proposed solution of the problem is the use of varying doping levels along the gain fiber to circumvent to mitigate the trade-off between thermal load and nonlinear effects.
\end{abstract}

\section{Introduction}

High- power fiber lasers (HPFL), originally developed for telecom applications [1], have numerous different applications in medicine, remote sensing, material processing etc. due to number of intrinsic advantages, including simplicity of optical cavity construction, high efficiency, excellent beam quality, and micro joule- level energies at high repetition rates that scale up processing speed [3], [7]. Although the pioneering works ware limited to sub-W levels [3], strong demand for higher and higher power levels have brought about rapid progress of technology resulted in development of multi-kW ones [10], [18].

A common approach for higher laser efficiency is to increase the dopant concentration which results in a higher absorption coefficient. Concurrently, this causes considerable thermal rise in the fiber core. Heating and beam-distorting effects are critical for high-power lasers [1]. Therefore, there is a great deal of interest in scaling average power along with heat removal and thermal management [12].

With regard to research, it can be said that previous solutions were focused on improvements of pumping diodes [3][5] and pumping technologies as well as fabrication and cooling process of gain fibers at different wavelengths [8][12]. In this context, important step forward in the evolution of the high gain fibers was introduction of cladding pumping [17]. Although the quantum efficiency double clad fiber lasers (DCFL's) is high, the temperature increase in the fiber core, generated by conversion of around $20 \%$ of pumping radiation into heat, will damage the coating material, which adversely affects the laser performance. So, ones more, it is necessary to emphasize importance of quantitatively describing and analyzing of thermal effects which strongly depend of the natural and geometrical properties of the fiber medium, pump beam intensity profile, and the laser cooling method used [12].

There is no doubt that the cooling process is very important but it is also quite certain that the process will be limited along increasing of expected power levels. Therefore, it may be deduced that it is needed to find some additive method to take heat under control. Herein doping management approach [18], revealed by Ilday and colleagues, can be promising method for further research.

This study is theoretical analysis of doping management, according to the thermal conductive equations taking to account natural and geometrical properties of the fiber medium as well as pump beam intensity profile.

\section{Temperature distribution of double-clad active fibers lasers}

Fiber temperature during operation is a $3 \mathrm{D}$ boundary value problem with the core acting as a heat source. The inner and outer claddings can be considered as one body since no source is represented in the cladding region resulting in secondary effects of outer cladding that can be ignored. The radial thermal distribution model for double-clad fiber is shown in Figure 1. $r$ is the radial coordinate and $\varphi$ is the tangential angle. The quantities $\boldsymbol{a}$ and $\boldsymbol{b}$ are core cladding radii, respectively [9]. $T_{1}(r, z)$ and $T_{2}(r, z)$ are the temperatures in the core and cladding regions, respectively.

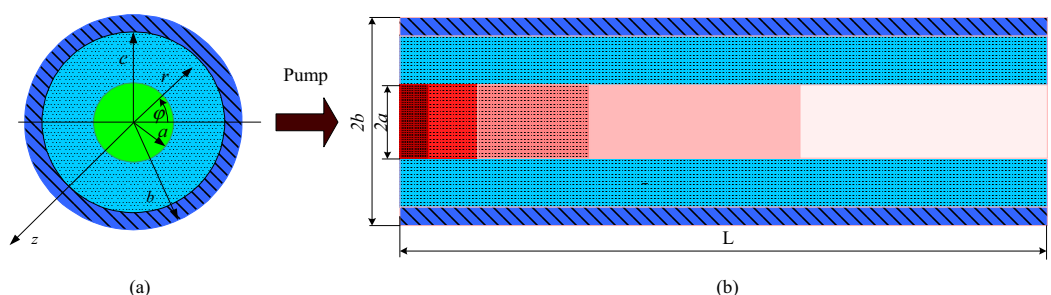

Fig. 1 Geometry of modeled double-clad fiber 
The stedy-state heat equations for both core and cladding regions are given as follows:

$$
\begin{aligned}
& \frac{1}{r} \frac{\partial}{\partial r}\left(r \frac{\partial T_{1}(r, z)}{\partial r}\right)+\frac{\partial^{2} T_{1}(r, z)}{\partial z^{2}}=-\frac{Q(r, z)}{k_{c}} ; 0 \leq r \leq a \\
& \frac{1}{r} \frac{\partial}{\partial r}\left(r \frac{\partial T_{2}(r, z)}{\partial r}\right)+\frac{\partial^{2} T_{2}(r, z)}{\partial z^{2}}=0 ; a \leq r \leq b
\end{aligned}
$$

In (1) $k_{f}$ denotes the thermal conductivity of the fiber and the heat density $Q(r, z)$ as functions of radius and propagation distance. For pumping distribution given by $P=\xi P_{o} \exp (-a(z) z)$ heat source density function can be represented as:

$Q(r, z)=\frac{\xi}{\pi a^{2}} P_{o} \exp (-a(z) z)$

where $P_{o}$ denote the pump power, $a(z)$ is the pump absorption coefficient, $\xi$ is the fractional thermal load or the conversion coefficient [14].

In (2) the right side of the equation is zero as no source is represented. Temperature functions from (1) and (2) have to be solved using method of separating variables with transformation $T(r, z)=\varphi(r) \theta(z)$, taking to account that temperature function has two separable components in radial and axial directions [12][13]. The differential equations to be solved are two separately differential equations with and without source respectively.

$$
\begin{aligned}
& \frac{1}{r \varphi_{1}(r)} \frac{\partial}{\partial r}\left(r \frac{\partial \varphi_{1}(r)}{\partial r}\right)+\frac{1}{\theta_{1}(z)} \frac{d^{2} \theta_{1}(z)}{d z^{2}}=-\frac{\xi P_{0} \alpha(z)}{k_{f} \pi a^{2}} \frac{\exp (-\alpha(z) z)}{\varphi_{1}(r) \theta_{1}(z)} \\
& \frac{1}{r \partial \varphi_{2}} \frac{\partial}{\partial r}\left(r \frac{\partial \varphi_{2}(r)}{\partial r}\right)+\frac{1}{\theta_{2}(z)} \frac{d^{2} \theta_{2}(z)}{d z^{2}}=0
\end{aligned}
$$

It is to be notified that both sides of equations (4) and (5) are divided by $\varphi(r) \theta(z)$.

As absorption coefficient is variable along of $z$, equation (4) is as such insolvable. Because of this, the fiber is considered as system divided into each $\mathrm{L} / \mathrm{n}$ length long $\mathrm{n}$ sub-systems along the fiber axis where $\mathrm{L}$ is the length of the fiber. And, for large values of $n$, all of that sub-systems act as separately part that absorption coefficient can be treated as constant. It is important to notify that, in terms of both power and temperature, the input of the each sub-system, except the first one, is the output of the previous one. So, pumping distribution of i-th sub-system, where $1 \leq i \leq n$ can be represented as:

$$
P_{i}=P_{(i-1)} \exp \left(-\alpha_{i} \frac{L}{n}\right)=P_{0} \exp \left(-\frac{L}{n} \sum_{j=1}^{i} \alpha_{j}\right)
$$

Taking to account this property, equations (4) and (5) can be rewritten as:

$$
\begin{aligned}
& \frac{1}{r \varphi_{1 i}(r)} \frac{\partial}{\partial r}\left(r \frac{\partial \varphi_{1 i}(r)}{\partial r}\right)+\frac{1}{\theta_{1 i}(z)} \frac{d^{2} \theta_{1 i}(z)}{d z^{2}}=-\frac{\xi P_{i} \alpha_{i}}{k_{f} \pi a^{2}} \frac{\exp \left(-\alpha_{i} z_{i}\right)}{\varphi_{1 i}(r) \theta_{1 i}(z)} \\
& \frac{1}{r \partial \varphi_{2 i}} \frac{\partial}{\partial r}\left(r \frac{\partial \varphi_{2 i}(r)}{\partial r}\right)+\frac{1}{\theta_{2 i}(z)} \frac{d^{2} \theta_{2 i}(z)}{d z^{2}}=0 \\
& \text { where } z_{i}=i \frac{L}{n} .
\end{aligned}
$$

For axial parts represented in exponential decay form $\theta_{1 i}(z)=$ $\exp \left(-\alpha_{i} z_{i}\right), \quad \theta_{2 i}(z)=\exp \left(-\beta_{i} z_{i}\right), \quad$ and $\quad \eta_{i}=$ $\xi P_{i} \alpha_{i} / k_{f} \pi a^{2}$ equations are transformed into modified zeroorder Bessel and zero-order Bessel equations respectively whose representations are given below:

$$
\begin{aligned}
& \frac{1}{r \varphi_{1 i}(r)} \frac{\partial}{\partial r}\left(r \frac{\partial \varphi_{1 i}(r)}{\partial r}\right)+\alpha_{i}^{2}=-\frac{\eta_{i}}{\varphi_{1 i}(r)} \\
& \frac{1}{r \varphi_{2 i}(r)} \frac{\partial}{\partial r}\left(r \frac{\partial \varphi_{2 i}(r)}{\partial r}\right)+\beta_{i}^{2}=0
\end{aligned}
$$

So, solutions for the radial parts of the temperature functions with and without source are:

$$
\begin{aligned}
\varphi_{1 i} & =a_{1 i} J_{0}\left(\alpha_{i} r\right)-a_{2 i} Y_{0}\left(\alpha_{i} r\right)-\frac{\eta_{i}}{\alpha_{i}^{2}} \\
\varphi_{2 i} & =b_{1 i} J_{0}\left(\beta_{i} r\right)-b_{2 i} Y_{0}\left(\beta_{i} r\right)
\end{aligned}
$$

Hence, the temperatures of $\mathrm{i}$-th sub-system can be represented as:

$$
T_{1 i}\left(r, z_{i}\right)=\eta_{i}\left[A_{1 i} J_{0}\left(\alpha_{i} r\right)-A_{2 i} Y_{0}\left(\alpha_{i} r\right)-\frac{1}{\alpha_{i}^{2}}\right] \exp \left(\alpha_{i} z_{i}\right)+T c
$$

$T_{2 i}\left(r, z_{i}\right)=\eta_{i}\left[B_{1 i} J_{0}\left(\beta_{i} r\right)-B_{2 i} Y_{0}\left(\beta_{i} r\right)\right] \exp \left(\beta_{i} z_{i}\right)+T c$ $A_{1 i}, A_{2 i}, B_{1 i}$, and $B_{2 i}$ are arbitrary constants to be determined from boundary conditions given below [10].

$\left.\partial T_{1}\left(r, z_{i}\right)\right|_{r=0}=$ finite

$\left.T_{1}\left(r, z_{i}\right)\right|_{r=a}=\left.T_{2}\left(r, z_{i}\right)\right|_{r=a}$

$\int_{V} Q\left(r, z_{i}\right) d V=\left.\int_{S} k_{c} \frac{\partial T_{2}\left(r, z_{i}\right)}{\partial r}\right|_{r=b} d S$

$\left.\frac{\partial T_{2}\left(r, z_{i}\right)}{\partial r}\right|_{r=b}=\frac{h}{k_{c}}\left(T_{c}-\left.T_{2}\left(r, z_{i}\right)\right|_{r=b}\right)$

$k_{c}, T_{c}$, and $h$ are the thermal conductivity, the cooling temperature, and the convective coefficient of the surface, respectively.

From conditions (15) and (16) it is found that $A_{2 i}$ have to be zero as well as $\alpha_{i}=\beta_{i}$. Hence, relation between $A_{1 i}, B_{1 i}$, and $B_{2 i}$ can be expressed as:

$A_{1 i}=B_{1 i}-B_{2 i} \frac{Y_{0}\left(\alpha_{i} a\right)}{J_{0}\left(\alpha_{i} a\right)}+\frac{1}{J_{0}\left(\alpha_{i} a\right) \alpha_{i}^{2}}$

Relation between $B_{1 i}$, and $B_{2 i}$ can be obtained introducing the definition for derivative of Bessel function in condition (17)

$B_{1 i} J_{1}\left(\alpha_{i} b\right)-B_{2 i} Y_{1}\left(\alpha_{i} b\right)=\frac{a^{2}}{2 b K \alpha_{i}}$

where $K=k_{c} / k_{f}$.

From condition (18) it is found that:

$B_{2 i}=\frac{h J_{0}\left(\alpha_{i} b\right)-k_{c} \alpha_{i} J_{1}\left(\alpha_{i} b\right)}{h Y_{0}\left(\alpha_{i} b\right)-k_{c} \alpha_{i} Y_{1}\left(\alpha_{i} b\right)} B_{1 i}$

Equations (19), (20), and (21) form an equation set whose solutions are found to be:

$A_{1 i}=\frac{a^{2}\left(J_{0}\left(\alpha_{i} a\right)-\gamma Y_{0}\left(\alpha_{i} a\right)\right)}{2 b K \alpha_{i} J_{0}\left(\alpha_{i} a\right)\left(J_{1}\left(\alpha_{i} b\right)-\gamma Y_{1}\left(\alpha_{i} b\right)\right)}+\frac{1}{\alpha_{i}^{2} J_{0}\left(\alpha_{i} a\right)}$

$B_{1 i}=\frac{a^{2}}{2 b K \alpha_{i}\left(J_{1}\left(\alpha_{i} b\right)-\gamma Y_{1}\left(\alpha_{i} b\right)\right)}$

$B_{2 i}=\frac{a^{2} \gamma}{2 b K \alpha_{i}\left(J_{1}\left(\alpha_{i} b\right)-\gamma Y_{1}\left(\alpha_{i} b\right)\right)}$

where $\gamma=\frac{h J_{0}\left(\alpha_{i} b\right)-k_{c} \alpha_{i} J_{1}\left(\alpha_{i} b\right)}{h Y_{0}\left(\alpha_{i} b\right)-k_{c} \alpha_{i} Y_{1}\left(\alpha_{i} b\right)}$

Finally, using equations (13-14) and (22-24), 2-D analytical solutions for temperature distribution at of core and clad regions respectively, for variable absorption coefficient, are obtained as follows:

$T_{1 i}\left(r, z_{i}\right)$

$=\frac{\xi \alpha_{i}}{k_{f} \pi a^{2}} P_{0} \exp \left(-\frac{L}{n} \sum_{j=1}^{i} \alpha_{j}\right)\left[\left(\frac{a^{2}\left(J_{0}\left(\alpha_{i} a\right)-\gamma Y_{0}\left(\alpha_{i} a\right)\right)}{2 b K \alpha_{i} J_{0}\left(\alpha_{i} a\right)\left(J_{1}\left(\alpha_{i} b\right)-\gamma Y_{1}\left(\alpha_{i} b\right)\right)}\right.\right.$

$\left.\left.+\frac{1}{\alpha_{i}^{2} J_{0}\left(\alpha_{i} a\right)}\right) J_{0}\left(\alpha_{i} r\right)-\frac{1}{\alpha_{i}^{2}}\right] \exp \left(-\alpha_{i} z_{i}\right)+T c$

$T_{2 i}\left(r, z_{i}\right)=$

$\frac{\xi \alpha_{i}}{k_{f} \pi a^{2}} P_{0} \exp \left(-\frac{L}{n} \sum_{j=1}^{i} \alpha_{j}\right)\left[\frac{a^{2}}{2 b K \alpha_{i}\left(J_{1}\left(\alpha_{i} b\right)-\gamma Y_{1}\left(\alpha_{i} b\right)\right)} J_{0}\left(\alpha_{i} r\right)-\right.$

$\left.\frac{a^{2} \gamma}{2 b K \alpha_{i}\left(J_{1}\left(\alpha_{i} b\right)-\gamma Y_{1}\left(\alpha_{i} b\right)\right)} Y_{0}\left(\alpha_{i} r\right)\right] \exp \left(-\alpha_{i} z_{i}\right)+T c$ 


\section{Results}

It can be said that the calculations are divided into 2 parts: verification of the functions for constant absorption coefficient and calculations for variable absorption coefficient. Table 1. shows the parameters used during the calculations.

\begin{tabular}{lll} 
Parameter & Amount & Unit \\
\hline $\mathrm{a}$ & 12.5 & $\mu \mathrm{m}$ \\
$\mathrm{b}$ & 125 & $\mu \mathrm{m}$ \\
$\mathrm{k}_{\mathrm{c}}$ & 23 & $\mathrm{~W} / \mathrm{mK}$ \\
$\mathrm{P}_{\mathrm{o}}$ & 150 & $\mathrm{~W}$ \\
$\mathrm{~T}_{\mathrm{c}}$ & 300 & $\mathrm{~K}$ \\
$\mathrm{~h}_{\mathrm{c}}$ & 50 & $\mathrm{~W} / \mathrm{m}^{2} \mathrm{~K}$ \\
$\mathrm{k}_{\mathrm{f}}$ & 230 & $\mathrm{~W} / \mathrm{mK}$ \\
$\mathrm{L}$ & 2 & $\mathrm{~m}$ \\
$\mathrm{n}$ & 400 & \\
$\lambda_{\mathrm{p}}$ & 976 & $\mathrm{~nm}$ \\
$\lambda_{\mathrm{p}}$ & 1060 & $\mathrm{~nm}$
\end{tabular}

Table1. Parameters used in calculations.

For constant absorption coefficient calculations 3 different absorption coefficients have been used: $\alpha_{1}=0.4, \alpha_{2}=0.1$, and $\alpha_{3}=0.01$. Fig. $2 . \mathrm{a}$ and $2 . \mathrm{b}$ show the temperature distributions of core and clad along $\mathrm{r}$ and $\mathrm{z}$ axis.

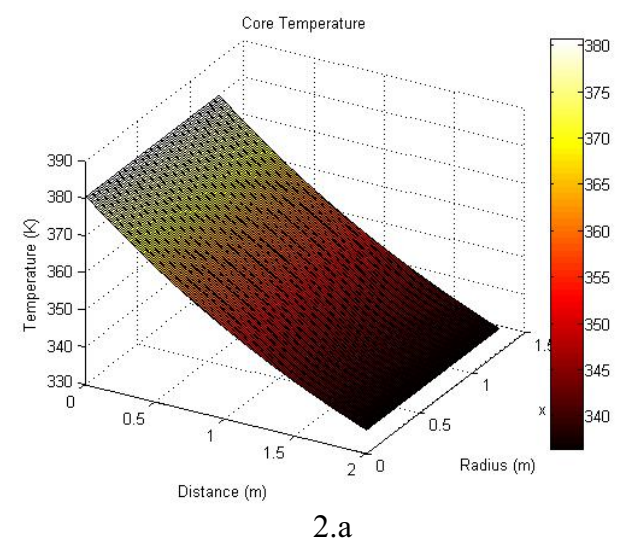

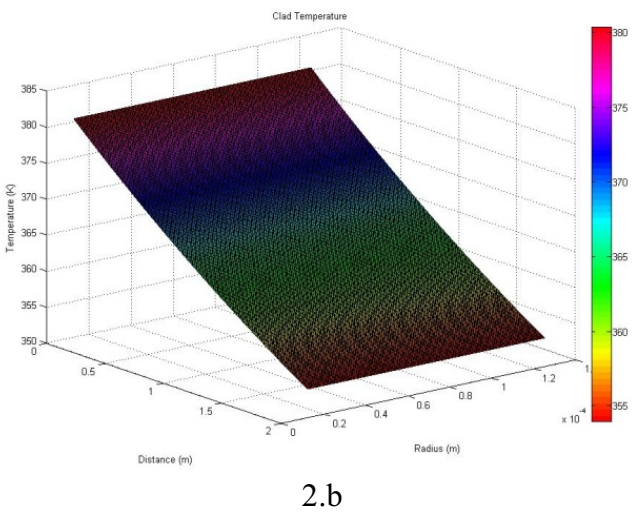

Fig. 2.a-b Illustrations of temperature distributions of core and clad respectively in r-z plane

In order to take more precise information the equations are plotted for both $\mathrm{r}=\mathrm{a}$ and $\mathrm{r}=\mathrm{b}$. One of these graphs is shown in Fig.3

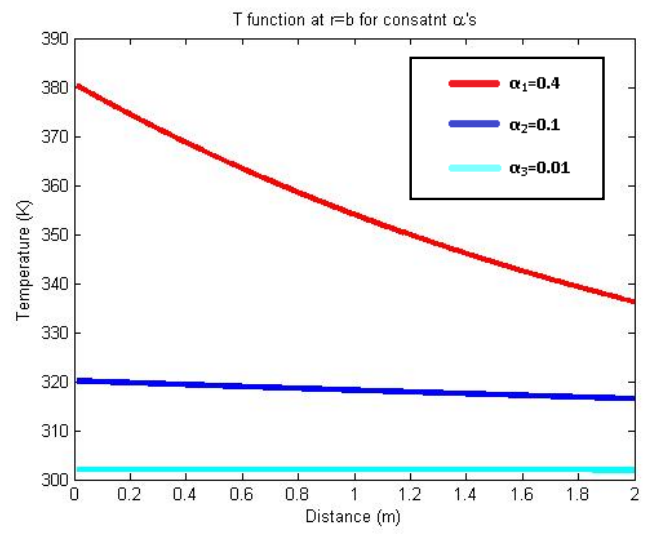

Fig.3 Temperature distribution at $\mathrm{r}=\mathrm{b}$

According to Figure 3 it is quite certain that an increase of absorption coefficient cases higher maximum temperature reached at pumping side of the fiber laser. At the same time, higher absorption coefficient provides more significant drop in temperature along the fiber. On this basis, it can be deduced that some increasing absorption function can cause reduction of the temperature along the fiber.

For calculations with variable absorption coefficient 3 different polynomials were used:

$\alpha_{1}(z)=-0.0081 z^{5}+0.0814 z^{4}-0.1628 z^{3}+0.1747 z^{2}-0.0237 z+0.01$

$\alpha_{2}(\mathrm{z})=0.0326 \mathrm{z}^{5}-0.179 \mathrm{z}^{4}+0.4036 \mathrm{z}^{3}-0.5651 \mathrm{z}^{2}+0.6221 \mathrm{z}+0.01$

$\alpha_{3}(\mathrm{z})=0.195 \mathrm{z}+0.01$

Figure 4.a illustrates 3 different increasing absorption coefficient functions given, rise between 0.01 and 0.4 , used for temperature calculations which results are represented in Figure 4.b. 


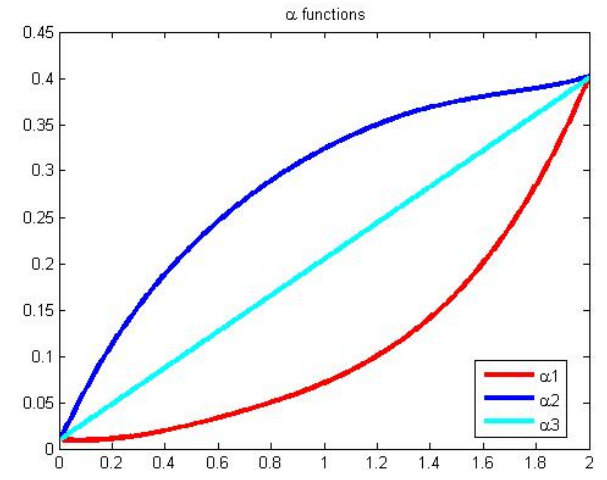

4.a

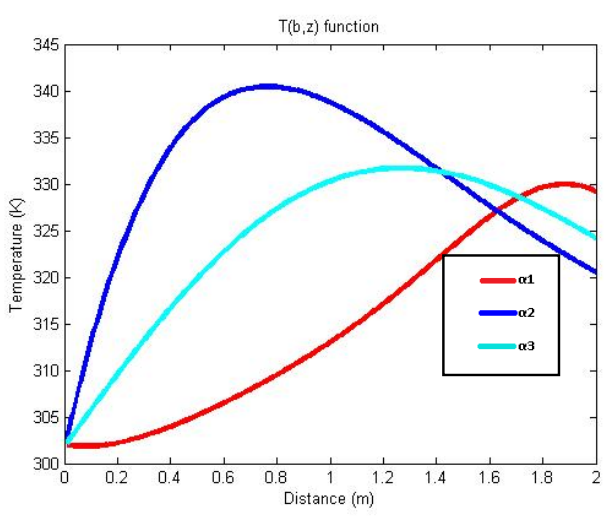

4.b

Fig.4.a-b Temperature distributions at $\mathrm{r}=\mathrm{b}$ for 3 different polynomials.

As can be seen from Figure 3.b gradual rise of the absorption coefficients results in gradual temperature increase. But, more important, all of the temperature functions with gradually raised absorption coefficient produce considerably smaller temperature rates then constant case, that verify practical results presented by Elahi and colleagues [18]. Additionally, the best results are achieved for the first absorption coefficient function.

\section{Conclusion}

In this work, at first we have constructed a heat dissipation model for double-clad fiber lasers with top-had pump beam with cooling at the outer cladding surface and analytically solved 3-D heat transfer functions for variable absorption coefficient. According to analysis, we have presented graphs for both different constant and different variable absorption coefficients. The calculations not only verify experimentally proved postulate of Elahi and colleagues but also show that gradually rise of absorption coefficient causes remarkable reduction in temperatures at core and clad of the fiber laser and may be lifesaver of devastating temperatures at fiber cores.

The numerical results also show that the third dimension in thermal analysis of any type of high-power fiber lasers cannot be ignored. Moreover, it can be introduced as active part of thermal control.

\section{References}

[1] Nilsson J., Sahu J.K., Jeong Y.: "High Power Fiber Lasers". Science 332, 921-922, 2011.

[2] Ueda K., Liu A.: "Future of High-Power Fiber Lasers". Laser Physics, 8/3 3, 774-781, 1998.

[3] Richardson D.J, Nilsson J., Clarkson W.A.: "High Power Fiber Lasers: Current Status and Future Perspectives". Optical Society of America B, B63-B92, 2010.

[4] Nilsson, J.: "High power fibre lasers: Exploitation of unique properties" Lasers and Electro-Optics and the European Quantum Electronics Conference. CLEO Europe - EQEC 2009.

[5] Lim X., Wang Y., Wang J.: "Development of Diode Lasers for Pumping High Power Ultrashort Pulse Lasers". Optical Fiber Conference, Optical Society of America, 27/11, USA 2010.

[6] Fermann M.E., Hartl I.:"Ultrafast Fiber Laser Technology". IEEE Journal of Selected Topics In Quantum Electronics, 15/1, 2009.

[7] J. Kirchhof, S. Unger, A. Schwuchow: "Materials for high-power fiber lasers". Journal of Non-Crystalline Solids, 352, 23-25, 2399-2403, 2006.

[8] Ueda K.: "High Power Fiber Lasers". CLEO/ Pacific Rim $4^{\text {th }}$ Conference of Lasers and Electro-Optics, 2001.

[9] Brown D.C.: "Thermal, Stress, and Thermo-Optic Effects in High Average Power Double-Clad Silica Fiber Lasers". IEEE Jurnal of Quantum Electronic 37/2, 2001.

[10] Assad M. E. H., Brown D. C.: "Thermodynamic Analysis of End-Pumped Lasers Subjected to Surface Cooling", IEEE Jurnal of Quantum Electronic 49/1, 2013.

[11] T. C. Newell, P. Peterson, and A. Gavrielides. M. P. Sharma: "Temperature Effects on the Emission Properties of Yb-doped optical fibers", Journal Article, Optical Society of America, 2006.

[12] Ashoori V, Malakzadeh A.: "Explicit exact threedimensional analytical temperature distribution in 
passively and actively cooled high-power fibre lasers", Journal Of Physics D: Applied Physics 44, 2011.

[13] Salim N. K., "An Overview of Heat Transfer Phenomena", ISBN 978-953-51-0827-6, InTech, 2012.

[14] Xue D.: "Three-Dimensional Simulation of the Temperature field in High-Power Double-Clad Fiber Lasers", Optics 122, 932-935, 2010.

[15] Li P., Zhu Ch., Zou S.: "Theoretical and Experimental Investigation of Thermal in High Power YtterbiumDoped Double-clad Fiber Laser" Optics and Laser Technology, 40237, 360-364, 2008.
[16] Canat G., Jaouën Y.: "Evidence of Thermal Effects in High Power $\mathrm{Er}^{3+}-\mathrm{Yb}^{3+}$ Fiber Lasers". Optics Letters, 30/22, 3030-3032, 2005.

[17] Müller H.-R., Kirchhof J., Reichel V.: "Fiber for HighPower Lasers and Amplifiers", Computer Rendus Physique 7, 154-162, 2006.

[18] Elahi P. Yılmaz S. Akçaalan Ö.: "Doping Menagement for High-Power Fiber Lasers: 100W, few-ps Pulse Generation from an All-Fiber-Integrated Amplifier". Optical Society of America, 2012. 